\title{
UNIVERSITYOF
}

FORWARD

THINKING

WESTMINSTER用

WestminsterResearch

http://www.westminster.ac.uk/westminsterresearch

\section{STAT5 in Cancer and Immunity}

Murphy, J. and Rani, A.

This is an author's accepted manuscript of an article published in the Journal of Interferon \& Cytokine Research, December 2015, ahead of print. doi:10.1089/jir.2015.0054.

The final publication is available from Mary Ann Liebert Inc. Publishers http://dx.doi.org/10.1089/jir.2015.0054

The WestminsterResearch online digital archive at the University of Westminster aims to make the research output of the University available to a wider audience. Copyright and Moral Rights remain with the authors and/or copyright owners.

Whilst further distribution of specific materials from within this archive is forbidden, you may freely distribute the URL of WestminsterResearch: ((http://westminsterresearch.wmin.ac.uk/)).

In case of abuse or copyright appearing without permission e-mail repository@westminster.ac.uk 


\title{
STAT5 in cancer and immunity
}

Aradhana Rani ${ }^{1}$ and John J Murphy ${ }^{1}$

${ }^{1}$ Department of Biomedical Sciences, University of Westminster, London, United Kingdom

Correspondence to: Aradhana Rani, A.Rani@westminster.ac.uk; John J Murphy, J.Murphy@westminster.ac.uk

\begin{abstract}
Signal transducers and activators of transcription 5 (STAT5a and STAT5b) are highly homologous proteins that are encoded by two separate genes and are activated by Janusactivated kinases (JAK) downstream of cytokine receptors. STAT5 proteins are activated by a wide variety of hematopoietic and non-hematopoietic cytokines and growth factors, all of which use the JAK-STAT signalling pathway as their main mode of signal transduction. STAT5 proteins critically regulate vital cellular functions such as proliferation, differentiation and survival. The physiological importance of STAT5 proteins is underscored by the plethora of primary human tumours that have aberrant constitutive activation of these proteins, which significantly contributes to tumour cell survival and malignant progression of disease.
\end{abstract}

STAT5 plays an important role in the maintenance of normal immune function and homeostasis, both of which are regulated by specific members of IL-2 family of cytokines, which share a common gamma chain $\left(\gamma_{c}\right)$ in their receptor complex. STAT5 critically mediates the biological actions of members of the $\gamma_{c}$ family of cytokines in the immune system. Essentially, STAT5 plays a critical role in the function and development of Tregs and consistently activated STAT5 is associated with a suppression in antitumour immunity and an increase in proliferation, invasion and survival of tumour cells. Thus, therapeutic targeting of STAT5 is promising in cancer. 
The Janus kinase (JAK)-signal transducer and activator of transcription (STAT) pathway represents an extremely rapid membrane to nucleus signalling system mediating cytokine signals in mammals $[1,2]$. It was studies on the interferon (IFN) receptor signalling that led to the discovery of the JAK-STAT pathway [3]. The JAK-STAT signalling is induced by engagement of a ligand (eg. cytokine) to its corresponding transmembrane receptor. This leads to dimerization of the receptor and thereby activation of receptor-associated JAKs, which subsequently phosphorylate tyrosine residues present in the cytoplasmic domain of the receptor. Phosphorylated tyrosine residues serves as docking sites for the src-homology2 (SH2) domains in STATs. The STATs are then phosphorylated by activated JAKs at a single tyrosine residue in the $\mathrm{C}$ terminus. Tyrosine-phosphorylated STATs form homo or heterodimers via reciprocal phosphotyrosine (pTyr)-SH2 interactions and are immediately translocated to the nucleus where they bind to palindromes of the general form TTC $\left(\mathrm{N}_{2}\right.$ ${ }_{4}$ )GAA, termed $\gamma$ activated sequences (GAS). The STATs are dephosphorylated by nuclear tyrosine phosphatases and exported to the cytoplasm to efficiently continue the phosphorylation-dephosphorylation cycle. Although recent studies on the STAT proteins have demonstrated constitutive energy and transport factor independent shuttling of STATs between the nucleus and cytoplasm [4,5], it is due to the specific conformation of tyrosine phosphorylated dimers that enables retention in the nucleus and the binding of STzATs to respective GAS sequences [4]. Recent studies have suggested a role for dephosphorylated STAT in the nucleus in maintaining the stability of transcriptionally repressed heterochromatin [6].

\section{STATs and protein structure}

The first STATs to be cloned by Darnell and co-workers were the IFN-inducible 91-kDa STAT1and the 113-kDa STAT2 proteins [3, 7]. The remaining five STATs were identified subsequently. There are seven STAT proteins in mammals, STAT1, STAT2, STAT3, STAT4, STAT5a, STAT5b and STAT6, with molecular masses between 75 and $95 \mathrm{kDa}$ and all are mapped to 3 chromosomal clusters being indicative of a common ancestral gene [8]. Out of the seven STATs, STATs 1, 3, 5A, and 5B can be activated by an array of cytokines including growth factors (EGF, PDGF, Hepatocyte growth factor, insulin-like growth factor, 
colony stimulating factor-1, Erythropoietin) and hormones (Prolactin, Growth hormone, Insulin) as well as downstream of some G-protein coupled receptors [1, 9, 10]. STATs 2, 4 and 6 are activated by a smaller subset of cytokines (IFN- $\alpha$, IL-12 and IL-4/IL-13 respectively).

All STAT proteins contain a conserved common structure containing the following domains: the $\mathrm{N}$ terminal domain (NTD), coiled-coil domain (CCD), src homology-2 (SH2) domain, linker domain (LD), DNA binding domain (DBD) and a transactivation domain (TAD) at the extreme C-terminus [11]. The conserved $\mathrm{N}$-terminal domain is involved in protein-protein interactions [12], and allows for cooperativity in dimer-dimer interactions and/or to form tetramers that can then bind to non consensus sites [13-15]. The NTD is conserved among the STATs, and it is $91 \%$ similar in the human STAT5a and STAT5b. Crystallographic studies on N-terminus of STAT4 revealed that it is composed of eight helices with a hooklike structure, which promote oligomerization of STAT dimers [16] and binding to tandemlylinked GAS sites $[14,17,18]$. Loss of cooperative STAT binding to tandem GAS sequences was observed due to mutation of a conserved tryptophan at position 37 to alanine (W37A), in STAT1, STAT4, STAT5a and STAT5b $[15,16]$, and furthermore in a loss of cytokineinduced phosphorylation of the critical tyrosine residue in the SH2 domain of STAT4 only [19]. Thus, the NTD serves a common function for all STAT proteins, which is to facilitate cooperative binding to tandemly-linked sites, and a selective role in the receptor-mediated activation of STAT4. Recently, an alternative more thermodynamically stable dimer interface for NTD interactions was determined, which identifies a conserved phenylalanine residue (F77 in STAT1, and F81 in STAT5) as being the critical residue [20]. As with W37A, mutation of this residue in STAT1 and STAT5 leads to a loss of tetramer, but not dimer formation on DNA [4]. Other functions of the NTD include its role in promoting proteinprotein interactions such as binding of $\mathrm{CBP} / \mathrm{p} 300$ to STAT1, receptor domains and PIAS family proteins $[19,21,22]$.

The CCD binds to other transcription factors and coactivators [16, 23] and is implicated in nuclear translocation and retention of STAT3 [24] as well as nuclear export of STATs [25]. The DBD, as the name suggests, mediates the binding of STATs to target sites with the canonical GAS sequence TTCN $_{3-4}$ GAA and defines the binding specificity [26-28], although the same is not the case with STAT2 [29]. Immediately following the DBD is a linker domain, which connects the DBD and the SH2 domain. The highly conserved SH2 domain 
interacts reciprocally with phosphorylated tyrosine residues on receptors, and thus has a role both in receptor docking and STAT dimerization [11]. The STAT family of proteins are phosphorylated at tyrosine and/or serine residues except STAT2, which is not known to be serine phosphorylated [30-32]. The variable TAD at the $\mathrm{C}$ - terminus is critical for transcriptional activation and interacts with additional co-factors [33]. The C-terminus of STAT1, STAT2, STAT5a, STAT5b and STAT6 interact with CBP/p300 [22, 34-36]. Recently it was proved that the DNA replication factor MCM5 which interacts with the Cterminus of STAT1 is essential for STAT1-mediated transcriptional activation [37]. STATs have also been shown to associate with AP1, IRF-1, NF-kB, Sp1, c-Jun, USF-1, Pu.1 and also with the glucocorticoid receptor [38-40].

\section{STAT5}

The transcription factor STAT5, was initially identified as a prolactin activated ovine mammary gland factor (MGF) [41]. Studies directed at IL-3 induced signal transduction and cloning, led to the discovery of two highly related isoforms, STAT5a and STAT5b [42, 43]. STAT5a is the major STAT5 isoform in the mammary gland, whereas STAT5b is the major STAT5 isoform in the liver. While STAT5a is mainly responsible for PRL dependent mammary gland development and function [44], STAT5b is required to maintain normal sexually dimorphic GH responses [45]. In humans, the genes for STAT5a and STAT5b map to chromosome 17q11.2, share 91\% homology at the amino acid level and differ primarily at their $\mathrm{C}$ terminus $[8,42,43,46]$. Obvious structural differences between the two forms of STAT5 include an additional 12 amino acids on the carboxy-terminus of STAT5a, which gives rise to a slightly shorter STAT5b and a 5 residue abbreviation of the STAT5a phosphotyrosyl tail segment between the SH2 and TAD domains.

\section{Modulation and regulation of STAT5 function}

STAT5 can be activated by a diverse group of cytokines, which include prolactin, growth hormone, erythropoietin (Epo), thrombopoietin (Tpo), granulocyte-macrophage colonystimulating factor (GM-CSF), epidermal growth factor (EGF), IL-2, IL-3, IL-5, IL-7, IL-9 and IL-15 [47, 48] and requires specific kinases (Table 1). The STAT5 signalling pathway is a transient and tightly regulated process, although less is known of the signals leading to its inactivation. The duration of activation of STAT5 is evident within minutes of cytokine 
stimulation and disappears a couple of hours later. However, it is found to be constitutively active in numerous primary human tumors, leukemias, and myeloproliferative disorders (MPDs) [49]. STAT5 function is regulated by post-translational modifications, members of SOCS family, PIAS family, caveolins, phosphatases and various protein-protein interactions.

STAT5 signalling is mediated by mechanisms that involve direct and indirect manipulation of STAT5 activity. STATs can be post-translationally modified by acetylation, ubiquitylation, glycosylation, ISGylation, sumoylation and the most common being phosphorylation [50, 51]. Post-translational modification can lead to STAT5 being either positively or negatively regulated.

\section{Tyrosine phosphorylation}

STAT5 is activated by tyrosine phosphorylation at position 694 and 699 for STAT5a and STAT5b respectively and can be negatively regulated by dephosphorylation. Tyrosine phosphosphorylation is crucial for STAT5 activity and can be phosphorylated by receptor associated JAKs as well as non-receptor Src kinases. Unlike JAKs, activation of STAT5 by Src kinases, leads to the translocation of only STAT5b into the nucleus [52]. Recent studies suggest a role for the src family of kinases (SFK)-STAT5 pathway in transformations and malignancies [53]. While a number of protein tyrosine kinases have been shown to phosphorylate STAT5, the phosphatases responsible for STAT5 dephosphorylation in-vivo remain elusive, despite a number of studies on candidate protein tyrosine phosphatases (PTP), including SHP-1, PTP1B, SHP-2, CD45, T-cell PTP (TC-PTP) and phosphatase 2A, which have been shown to dephosphorylate STATs in vitro [54-58]. Overexpression of PTP1B leads to dephosphorylation of STAT5 [57]. SHP-2 is a STAT5a phosphatase involved in dephosphorylation of the tyrosine-phosphorylated STAT5a in the cytoplasm [20]. Recently it has been shown that the small dual-specificity phosphatase "VHR (Vaccinia H1 Related)" dephosphorylates tyrosine-phosphorylated STAT5, subsequently inhibiting STAT5 function [59]. The phosphorylation of VHR is required to activate its phosphatase activity toward STAT5 [59]. The possibility of other unidentified nuclear phosphatase(s) that dephosphorylates STAT5 still exists. Nevertheless, phosphorylated STAT5 proteins are known to be targeted for degradation via the ubiquitin-mediated proteasomal degradation pathway $[60,61]$. 


\section{Serine phosphorylation}

In addition to tyrosine phosphorylation, STAT5a and STAT5b are also induced to undergo serine phosphorylation [1]. Phosphorylation of serine residues is independent of tyrosine phosphorylation. Mutational studies on mouse Stat5 genes and the development of phospho specific antibodies helped to identify serine phosphorylation sites in a conserved PSP motif of Ser725 for STAT5a and Ser730 for STAT5b [62]. Serine phosphorylation at the S725 and S779 residues of STAT5a cooperate to negatively regulate PRL-induced transcription of $\beta$ casein in the absence of costimulation of the glucocorticoid receptor [63]. The biological and physiological implications of STAT5 serine phosphorylation are not completely understood, though a study on expression of p21-activated kinase (Pak1), a serine/threonine protein kinase, showed that it associates with STAT5 and phosphorylates STAT5a at serine position S779 and thereby stimulates $\beta$-casein promoter activity [64]. Clark et al have identified a novel STAT5a serine phosphorylation at S127/128 which is critical for ERBB4-induced STAT5a stimulation and phosphorylation at S779 is regulated by ERBB4 expression in mammary glands [65].

Friedbichler et al in 2010 demonstrated that serine phosphorylation plays an improtant role in leaukemogenesis [66]. More recently, it has been shown that serine phosphorylation leads to nuclear transport of STAT5 in BCR-ABL induced disease (chronic myeloid leukemia) [67].

\section{Glycosylation}

STAT5 can be post-translationally modified by glycosylation. A study on HC-11 mammary epithelial cells has reported glycosylation of STAT5a by O-linked N acetyglucosamine (OGlcNAc) following STAT5 activation and the glycosylated form is mainly found in the nucleus of hormone-induced cells [68]. The glycosylation occurs at the $\mathrm{N}$ - terminal threonine T92 position [68]. A mutation at T92 led to loss of STAT5 glycosylation and thereby its ability to bind the transcriptional coactivator CBP. Studies done by Nanashima et al went onto demonstrate that STAT5a was modified by O-GlcNAc in the Hirosaki hairless rat (HHR) and was identified in the nucleus [69]. Additionally, the glycosylated STAT5a was shown to bind to the STAT5-responsive element with an enhanced affinity. 


\section{SUMOylation and PIAS}

SUMO is an ubiquitin-related molecule and protein-SUMO conjugation (Sumoylation) has been found to have various functions, including positive and negative regulation of STAT transcriptional activity. There are three forms of SUMO peptide, namely SUMO1, SUMO2 and SUMO3, and they consist of around 100 amino acids, which are added to a consensus sumoylation site ( $\Psi \mathrm{KXE})$. SUMOylation is catalyzed by SUMO-specific E1, E2, and E3 ligases and can be reversed by SUMO-specific proteases (SENPs). Sumoylation has been shown to modify protein function by altering the function, localization and extent of ubiquitination [70]. ICA512 which is a catalytically inactive member of the receptor protein tyrosine phosphatase (PTP) family, mediates the binding of PIASy to STAT5 and sumoylation of ICA512 regulates its binding to STAT5 [71]. Recently, Van-Nguyen et al have shown that the activity of STAT5 is inhibited upon active SUMOylation of STAT5. This was due to an absence of SENP1 and thus, SENP1 played a role in the SUMOylation and regulation of STAT5 transcription during lymphoid development [72].

Originally discovered as negative regulators of STAT signalling, the mammalian protein inhibitors of activated STATs (PIAS) family consists of 5 members, PIAS1, PIAS3, PIASx $\alpha$, PIASx $\beta$ and PIASY [21]. Although several molecular mechanisms have been proposed to explain transcriptional regulation by PIAS proteins, the ones of interest for STAT5 are: PIAS may repress transcription by inhibiting the DNA-binding activity of STAT5 or PIAS may regulate transcription by promoting sumoylation of STAT5. Thus, it was observed by Rycyzyn and Clevenger, that overexpression of PIAS3 in CHO cells represses STAT5 transcriptional activity [73]. Recently, a functional role for the E3 ubiquitin ligase c-Cbl was demonstrated in differentiation of osteoblasts and that blocking c-Cbl activity, downregulates STAT5 ubiquitination and promotes bone regeneration [74].

\section{Transcriptional co-activators of STAT5}

A number of proteins are known to interact with STAT family members and interaction of STAT5 with additional proteins including transcription factors and co-activators regulates its activity. Transcriptional initiation requires the interaction of STAT5 with the initiation complex in addition to co-regulatory proteins. These co-activators mainly interact with the 
TAD of STAT5, although they are also known to interact via the CCD and SH2 domains. The interaction of STAT5 with other nuclear factors presents a regulatory mechanism that determines greater potency of signalling and/or greater selectivity of target genes [35]. One of the first studied factors was CBP which is the binding protein for cAMP response element binding protein (CREB). CBP and p300 interact with the TAD of STAT5 [75]. Nuclear receptor co-activator 1 (NCoA-1) binds to the TAD domain of STAT5a and is essential for the transcriptional activity of STAT5a [76]. N-myc interacting protein (Nmi) is another STAT5 interacting protein that augments the recruitment of CBP/p300 to STAT5 [35]. The glucocorticoid receptor (GR) has been reported to interact with the N terminus of STAT5 and is required for many functions exerted by either transcription factors [77]. GR binds to STAT5a and has a physiological significance in the expression of milk protein genes [78]. A list of some of the known protein interactions with STAT5 are listed in Table 2.

\section{Interaction of STAT5 to DNA binding sites}

The STAT5 proteins interact directly with specific DNA elements through the DNA binding domain in the center of the STAT5 structure along with cooperation of all the other domains. The SH2 domain of STAT plays a vital role in the dimerization [79], and gene regulation of STAT5 may be influenced by the pattern of STAT5a and STAT5b pairing as homo or heterodimers [80-82]. It is known that DNA binding requires dimerization, which is mediated by phosphotyrosyl-SH2 domain interactions between two STAT5 proteins. STAT5 forms supramolecular complexes (dimer-dimer or higher) on target sites containing two or more neighbouring STAT binding sites $[4,15]$.

STAT dimers can undergo tandem linkage through their $\mathrm{N}$ terminal domains when bound to closely spaced GAS sites [17]. STAT5a and STAT5b exhibit differences with respect to their tissue distribution [42, 43]. Park and Waxman in 2001 were able to prove that heterodimerized (STAT5a-STAT5b) and homodimerized (STAT5b-STAT5b) STAT5 complexes play distinct roles in the sexually dimorphic responses of the liver to growth hormone [83]. It has previously been reported that cytokines cause tyrosine phosphorylation and DNA binding of predominantly one STAT5 protein (STAT5a) despite ample expression of both forms of STAT5 in certain cell contexts [84]. One study reported different DNA binding specificities for STAT5a homodimers versus that of STAT5b homodimers due to a single amino acid change in DNA binding domains of the two proteins [85]. However, generally there are no significant differences in DNA binding specificity between STAT5a 
and STAT5b [86-88]. The consensus STAT5a and STAT5b binding sites that have been defined in vitro are TTCYNRGAA and TTC(T/C)N(G/A)GAA [86, 89]. The STAT5 alignment matrix prepared by Fung et al also favours the consensus GAS motif TTC(T/C)N(G/A)GAA [90].

Previous studies have established a role of the $\mathrm{N}$ terminal domain of STAT proteins in formation of tetrameric complexes $[16,18]$. STAT5 tetramer formation does not require high affinity sites, and binding of STAT tetramers to weak binding sites produced even more stable complexes than single dimers bound to high affinity sites [15, 16]. Purified STAT5a has a higher DNA binding affinity and could bind to chromatin in the tetrameric form compared to STAT5b which preferentially bound to chromatin as dimers [86]. Tetramer competent STATs could have a wider range of potential DNA binding sites $[15,86]$. The importance of tetramerization of STAT5 was shown for the transcriptional activation of CD25 promoter $[4,15]$. Tetramerization of STAT5 has been associated with leukemogenesis [91]. A constitutively active STAT5a mutant (cS5F) increased the abundance and stability of STAT5 tetramers compared to the unmutated form and $\mathrm{N}$ terminal mutations directed at only tetramer formation failed to induce leukaemia, suggesting that tetrameric STAT5 complexes may regulate a different subset of target genes some of which drive tumorigenesis [91]. A recent study, in STAT5a-Stat5b double knock-in (DKI) mice that form dimers but not tetramers, identified genes regulated by STAT5 tetramers, in addition to defining the consensus sequences required by dimers versus tetramers [92]. The study shows that tetramerization is critical for normal immune function.

\section{BIOLOGICAL FUNCTIONS OF STAT5}

Despite sharing $~ 96 \%$ homology at the protein level, both Stat5 proteins have overlapping and distinct functions. Generation of single and double KOs and transgenic mice for STAT5a and Stat5b proteins has greatly enhanced the understanding of the biological roles of these proteins. Indeed, most studies investigationg the role of STAT5 in immunity were performed on the "STAT5 null mice" that still retained a residual protein lacking the N-terminal domain of STAT5, also denoted as the STAT5a/b ${ }^{\Delta \mathrm{N} / \Delta \mathrm{N}}[44,93,94]$. However, more recent data have been derived from the analysis of STAT5 null mice presenting with a complete deletion of the STAT5a/b gene locus, also denoted as STAT5a/b fl/fl, lck-cre and the STAT5a/b null/null 
mice. The difference between the mouse models STAT5a/b $\Delta \mathrm{N} / \Delta \mathrm{N}$ and STAT5a/b null/null are strong and implies classified roles for the $\mathrm{N}$ terminal truncated STAT5.

\section{Role of STAT5 in immunity}

STAT5a/b are crucial regulators of the immune system. An initial understanding of its role came through knock out mice generated in 1998 which was enhanced upon, by the next generation of null mice generated a few years later in 2004 . Thus, the implications of the role of STAT5 are derived from two generations of STAT5 ${ }^{\Delta \mathrm{N}} /$ null mice $[94,95]$.

\section{Studies from the first generation of KO mice (STAT5a/b $\left.{ }^{\Delta N / \Delta N}\right)$}

Most studies that investigated the role of STAT5A/B in lymphopoiesis employed the STAT5A/ ${ }^{\Delta N}, S_{T A T} 5 B / \Delta N$ and the STAT5A/B ${ }^{\Delta N / \Delta N}$ mice. These STAT5A/ ${ }^{\Delta N}$ and STAT5B/ $/ \Delta N$ mice (as well as $\mathrm{STAT} 5 \mathrm{~A} / \mathrm{B}^{\Delta \mathrm{N} / \Delta \mathrm{N}}$ mice) were viable and revealed surprisingly mild phenotypes in the development and function of $\mathrm{T}$ and $\mathrm{B}$ lymphocytes. The phenotype of the STAT5A/B ${ }^{\Delta N / \Delta N}$ mice was expectedly, more severe than either of the single KOs $(\mathrm{STAT} 5 \mathrm{~A} / \Delta \mathrm{N}$ or $\mathrm{STAT} 5 \mathrm{~B} / \Delta \mathrm{N})$ alone, and not only showed reduced proliferation of splenocytes but also splenomegaly along with reduced number of NK cells and activated phenotype of T cells [96].

Upon studying the lymphoid development in STAT5A/B ${ }^{\Delta N / \Delta N}$ double mutant mice, there was a subtle reduction in $\mathrm{T}$ and $\mathrm{B}$ cell numbers, which was accompanied by a complete lack of natural killer $(\mathrm{NK})$ cells and $\mathrm{CD} 4+\mathrm{CD} 25+$ suppressor T cells.

STAT5A/ ${ }^{\Delta N}$ deficient $T$ cells presented a reduction in the expression of cytokine receptor IL$2 \mathrm{R} \alpha$ and this was confirmed by the finding that its expression was upregulated by the cytokine IL-2 [97]. Studies on STAT5A/ ${ }^{\mathrm{N}}$ and STAT5B/ ${ }^{\Delta \mathrm{N}}$ mice demonstrated defects in T cell proliferation and function [98]. While splenocytes from STAT5A/ ${ }^{\Delta \mathrm{N}}$ mice showed partial impairment in IL-2 mediated proliferation, STAT5B/AN mice showed even more severe defect in proliferation $[98,99]$. STAT5A/B ${ }^{\Delta N / \Delta N}$ mice caused impaired proliferation in response to IL-2 and halted cell-cycle progression to mature T cells [94, 96, 100]. Both Stat5 proteins regulate TCR-mediated proliferation of CD4 T cells [96], and a greater defect was observed in STAT5B/ ${ }^{\Delta N}$ than in STAT5A/ ${ }^{\Delta N}$ mice with regard to reduced number of natural killer (NK) cells, suggesting that STAT5a and STAT5b play an unequal role in normal NK 
cell development [96]. NK cells are absent in STAT5A/B $\mathrm{B}^{\Delta \mathrm{N} / \Delta \mathrm{N}}$ mice and there is no cytotoxic activity in presence or absence of IL-12 or IL-15 [96]. A role for STAT5 in the survival and differentiation of memory CD8 T cells was confirmed by studies using the STAT5A/ $\triangle \mathrm{N}$ and STAT5B $/ \Delta \mathrm{N}$ mice, which had reduced numbers of $\mathrm{CD} 8+\mathrm{T}$ cells from splenocytes and transgenic expression of STAT5 resulted in an increase in the number of CD8+ T cells [101].

It was studies on allergic late-phase reactions that led Kagami et al to demonstrate that STAT5A $/ \Delta \mathrm{N}$ and $\mathrm{STAT} 5 \mathrm{~B} / \Delta \mathrm{N}$ mice had reduced $\mathrm{CD} 4+\mathrm{T}$ cell infiltration along with diminished eosinophil recruitment. This was due to defective STAT5 functions in these mice, leading to a reduction in antigen mediated proliferation of splenocytes and $\mathrm{T}$ cells and a subsequent decline in the antigen induced $\mathrm{T}$ cell infiltration in the airways [102]. The role of STAT5 in T helper cell differentiation was defined on studies using the STAT5A/ ${ }^{\Delta N}$ mice. Th2 cell differentiation from antigen-stimulated splenocytes was significantly decreased in STAT5A/ $\triangle \mathrm{N}$ mice suggesting that STAT5a regulates $\mathrm{T}$ helper cell differentiation. The impairment in Th2 cell differentiation was detected in the presence of high concentrations of IL-4, which was restored upon retrovirus-mediated expression of STAT5b. Although Friedrich et al noted that STAT5b is a mediator of IL-4 induced cell proliferation [103], a role for IL-4 mediated activation of STAT5 was dismissed due to no phosphorylation of STAT5a even in the absence of STAT6. There was also an impairment in the development of Tregs $(\mathrm{CD} 4+\mathrm{CD} 25+)$ in the STAT5A/ ${ }^{\mathrm{N}}$ mice and a depeletion of the Tregs from spelenocytes of these mice did not impair differentiation into Th1 or Th2 cells. Thus, STAT5a is essential for the differentiation into Th2 and development of Tregs.

Additionally, mice deficient in STAT5a/5b demonstrated reduced numbers of peripheral B cells and of B-cell precursors in the bone marrow [104].

\section{Studies from the second generation of STAT5 KO mice (STAT5a/b null/null)}

Most recent data have been derived from the analysis of STAT5-null mice, in which the entire STAT5a/5b locus was flanked by LoxP sites and further deleted by the CRE-mediated recombination (using the Cre/Lox technology) [95]. The differences between the two mouse models (STAT5A/B ${ }^{\Delta \mathrm{N} / \Delta \mathrm{N}}$ and STAT5a/b null/null $)$ are strong. For instance, STAT5a/b null/null lack CD8+ T lymphocytes and failed to develop T, B and NK cells [105, 106]. Moreover, Bcell development is abrogated at the pre-pro B cell stage in the bone marrow of STAT5a/b 
null/null mice when compared to the previous truncated version of STAT5A/B/ $/ \Delta \mathrm{N} / \Delta \mathrm{N}$ mice. These studies lead to an important conclusion of the differences between the two generations of KO mice. The truncated versions of STAT5 proteins expressed in the STAT5A/B/ $\triangle \mathrm{N} / \Delta \mathrm{N}$ mice are able to compensate and to a large extent not only resucue B cell development, but also support the development of CD8+ T cells. It is now well known that the truncated versions of STAT5 can bind to a fraction of the STAT5 target genes within the nucleus, thereby activating or repressing them.

The first evidence of the role of STAT5 in CD8+ T cell differentiation came with studies on STAT5b-CA mice which exhibited increased numbers of CD8+ T lymphocytes. This was confirmed when Park et al, proved, that strong TCR signals led to a repression in the IL-7R which subsequently led to a downregulation of CD8 expression [107]. According to the coreceptor model, high affinity TCRs differentiate into CD4+ T cells while low affinity TCR's lead to differentiation and expression of CD8+ T cells. Interestingly, the molecular basis of this CD8 T cell differentiation was due to a dependance on STAT5 signalling and was determined by expressing Runx3, the master regulator for CD8 T cells [108]. Thus, STAT5 signalling is required in mature $\mathrm{CD} 8+\mathrm{T}$ cells for the expression of the CD8 master regulator Runx3 and CD8 .

It was Zhu et al, who in 2003 first suggested a role for STAT5 in Th2 differentiation, by ectopically expressing activated STAT5[109]. This led to a skewing of the cells from Th1 to Th2 fate. These studies demonstrated that expression of STAT5 ectopically, suppressed Th1 and Th17 differentiation, while promoting Th2 cell differentiation by suppressing the production of IFNy. Thus, both STAT5a and STAT5b regulate Th2 cell differentiation. In brief, IL-2 regulates Th2 cell differentiation by upregulating GATA3 and an upregulation in the IL-4R due to the binding of STAT5 to the promoter of the $I L 4 r$ [110]. IL-2 via STAT5 augments and regulates the expression of IL12rb2 and Tbx21, leading to an enhancement in Th1 responses as well [111].

Previous work on the STAT5A/B ${ }^{\Delta N / \Delta N}$ mice had demonstrated that Treg numbers were reduced in these mice and that activation of STAT5 led to an increase in Tregs. However, further work using the STAT5a/b null/null mice established that conditional deletion of STAT5 in double positive thymocytes, resulted in decreased numbers of $\mathrm{CD} 8^{+} \mathrm{T}$ and a failure to develop into Tregs [105]. Most importantly, none of the surviving tregs had the deleted STAT5 protein, which goes on to support its role in the development of Tregs. This 
observation was confirmed using STAT5b-CA mice lacking the IL-2Rb, which suggested that STAT5 played a crucial role in rescuing Treg development in the IL-2Rb negative mice. At the same time, these studies also went onto reveal the binding sites of STAT5 on the Foxp3 promoter, which is master regulator of Tregs [112].

\section{Role in haematological malignancy}

STAT5 activation has been found in blood malignancies (HTLV-1 dependent leukaemia, Erythroleukemia, Acute lymphocytic leukaemia (ALL), Acute myelogenous leukaemia (AML), Chronic myelogenous leukaemia (CML), megakaryocytic leukaemia (ML) and Hodgkins lymphoma [113-116] and human tumours (breast, prostate, ovary, head and neck cancers) $[117,118]$. STAT5 has also been shown to be constitutively activated in cutaneous lymphomas, while dysregulated expression of a C-terminally truncated form of Stat5 in Sezary Syndrome was associated with a loss of IL-2-induced gene expression [119]. Although both the STAT5 isoforms have been involved in human cancers and tumors, the exact role of each isoform in a specific cancer has not yet been illustrated.

With the prominent role played by STAT5 in the development, differentiation and survival of lymphoid cells, it is no wonder then that it is involved in hematologic malignancies. ALL is a neoplastic disease of both children and adults characterised by acquired genetic alteration, chromosomal alterations and translocations are factors that lead to the diagnosis and prognosis of the disease. Studies on the ABL oncogene demonstrated that the STAT5a/b null/null cells are refractory to transformation by the Abelson oncogenes and subsequently fail to induce lymphoid leukemia, whereas $\mathrm{STAT} 5 \mathrm{~A} / \mathrm{B} /{ }^{\Delta \mathrm{N} / \Delta \mathrm{N}_{-}}$-derived cells are readily transformed [105]. Multi-lineage leukemia was also observed in mice expressing the constitutively active STAT5a/b which was due to the tetramerization capability of STAT5a/b in these mice [91]. Thus, although the truncated version of STAT5 is deficient in the tetramerization domain, they are able to compensate for the loss by binding to oncogenic tyrosine kinases (eg ABL oncogene). Xia et al, demonstrated that the naturally occurring truncated forms of STAT5 were responsible for blood cell cancers and was the main cause for the relapse of the cancer [120]. The role of STAT5 in ALL was confirmed when, Nakayama et al, found that STAT5b-CA mice developed symptoms closely related to human pre-B ALL [121]. Another set of studies also demonstrated that a transgenic STAT5-CA 
mouse presented with loss of p53 and concurrent initiation of B cell leukemia [122]. Lessons learnt from a limited study of human samples also supported the evidence and that STAT5 was constitutively activated in more than $60 \%$ of frozen ALL samples [123, 124].

A classic example of the complex nature of the cascade of signalling pathways that can be activated by a single oncogenic protein in cancers is that of signalling mediated through the Bcr-Abl tyrosine kinase, which also induces constitutive STAT5 activation in CML [117]. Studies on mice suggested that Stat5 was not required for the induction of BCR-ABLinduced CML-like leukemia [104]. In contrast, recent studies showed that STAT5 is required for the development of leukemia upon introduction of BCR-Abl [105]. In the absence of STAT5a, the incidence of CML was reduced [125]. Serine phosphorylation of STAT5 is essential for leukemogenesis and serine phosphorylation of STAT5a is necessary for nuclear localization of STAT5 in BCR-Abl ${ }^{+}$cells and that formation of tetramers rather than dimers is associated with leukemogenesis [66,91]. Furthermore, a recent study demonstrated that BCR-ABL1 aftects STAT5A/B differentially. Using a BCR-ABL positive cell line, STAT5B RNAi knock down led to sensitization of leukemic cells to treatment by imatinib [126], and STAT5A attenuation enhanced the basal oxidative stress and DNA damage of normal CD34 positive and CML cells. Attenuation of STAT5a also resulted in the inhibition of growth in CD34 positive CML cells from imatinib resistant patients [127].

CML is a clonal hematopoietic stem cell (HSC) malignancy characterized by chromosomal translocation and the hallmark oncogenic event is the formation of the mutant BCR-ABL and activation of STAT5 [115]. Indeed, BCR-ABL1 is crucial for the survival and proliferation of leukemic cells in chronic stage CML and Hochhaus et al showed that BCR-ABL1-targeting drugs have posititive response in many patients with Philadelphia chromosome positive $(\mathrm{Ph}+)$ CML [128]. Imatinib was one of the most successful drugs with therapeutic implications in leukemia. Dasatinib is another drug with huge implications in leukemia.

Onishi et al identified and characterized a constitutively active STAT5 mutant that induces certain properties of malignant cells [129] and studies have identified the cause of the constitutive activation as being dependent on its specific role in a specific malignancy. Interestingly, constitutive activation of STAT5 is at times due to its induction by constitutively active JAK2. Indeed, the constitutive activation of STAT5 in some 
myeloproliferative disorders is due to the JAK2 V617F mutation [130, 131]. And constitutive activation of STAT5 is also observed in myelodysplastic syndrome (MDS) and in myeloproliferative diseases, such as polycythemia vera (PV), where there is associated dysregulation of the upstream activating kinases, JAK1 or JAK2 [132, 133]. However, in AML, constitutive activation of STAT5 is driven by FLT3. FLT-3 and $c$-KIT gene mutations lead to STAT5 activation in leukemias as noted in these studies [134, 135]. Thus, activated FLT3 has the potential to transform hematopoietic cells and activates STAT5 in primary AML cells.

Thus, a STAT5a/b targeting molecule in combination with other therapeutic agents as well as imatinib/dastinib could be used in leukemia.

\section{Role in breast cancer}

The STAT5 proteins are not only critical in hematopeoitic malignancies, but also in several other cancers and tumours. It presumably plays complex opposing roles in breast cancer (BC) and summarised below are the studies in breast cancer cell lines, animal models of breast cancer as well as human BC samples.

In studying the role of STAT5 in breast cancer, it is only justified to enlist that STAT5 plays an important role in proliferation, survival and terminal differentiation of the mammary gland $[95,136,137]$. These functions are made possible by upregulating the pro-survival genes (bcl-xl), as well as genes for proteins found in milk, beta-casein and whey acidic protein [95, 138, 139]. STAT5 is a promoter of tumorigeneis in rodent mammary gland and the presence of STAT5a is required for the development of oncogene-induced mammary cancers in mice [140-142]. Knockout studies on mice indicate that a loss of STAT5a delays tumorigenesis and hemizygous loss of the STAT5a allele led to a delay in tumours initiation and formation $[141,142]$. Additionally, constitutive activation of STAT5a or over expression of STAT5 led to the mammary tumours within 8-12 months [143]. In this study, it was noted that truncated STAT5 (carboxy terminal truncation) overexpression led to a poorly differentiated mammary tumour. Thus, it could be speculated that the poor differentiation was due to the wtSTAT5 being inhibited and that the carboxy terminal truncated STAT5 plays an important role in malignancy and tumour formation. Again, Vafaizadeh et al, using a lentiviral infection system, created a constitutively active STAT5 (cS5-F) which upon expression, led to 
epithelial hyperproliferation and ER+ PR+ adenocarcinomas [144]. Nonetheless, STAT5 also has a protective role in BC cells and although STAT5B is constitutively active in human BC cell lines, over expressing a dominant negative variant of STAT5 leads to an induction of apoptosis in luminal BC cell lines [145]. Recent work by Caffarel et al, using the constitutive active JAK2-V617F mutant and expressing it in MCF-7 BC cells, led to a more invasive form of BC in xenografts [146].

Thus, while knockout studies on mice and BC cell lines indicate that a loss of STAT5 delays tumorigenesis, this is not the case in humans. Infact, one of the most notable findings from clinical studies was done using immunohistochemistry to the tyrosine phorpshoryakted protein and it was observed that while $40 \%$ of all breast cancers displayed activation of STAT3 alone, only $7 \%$ of them showed STAT5 activation. Of the tumors that presented with activated STAT5, more than $80 \%$ had activation of STAT3 as well. Furthermore, an analysis of the pathological features of these tumors revealed that cancers displaying activation of STAT5 were more likely to be highly differentiated, low grade tumors. Additionally, they were less likely to form metastases to lymph nodes. All of these properties of tumors with activation of both STAT3 and STAT5 reflected a more favorable clinical outcome than tumors displaying activation of STAT3 alone. Indeed, patient survival was significantly prolonged when tumors displayed gene expression signatures consistent with activation of STAT3 and STAT5 rather than STAT3 activation alone [147]. In another study by Yamashita et al, STAT5 was defined as a strong prognostic molecular marker in ER-positive breast cancer. In this study, they studied the expression of STAT3 and STAT5 in more than 500 breast cancer tissues by immunohistochemical techniques and observed that in ER positive patients having Stat5 positive tumours had significantly increased overall survival, thereby suggesting that expression of Stat5 is helpful in selecting patients who could possibly benefit from endocrine therapy [148]. These findings thus correspond to the notion that normally STAT5 promotes differentiation of mammary epithelium. Moreover, they also suggest that tumors with activation of STAT5 (or both STAT3 and STAT5) may be more susceptible to cell death induced by chemotherapeutic agents, which could also contribute to a more favorable prognosis. Various other studies go onto define the protective role of STAT5 in breast cancer pathogenesis. STAT5 expression and activation when studied in breast cancer samples showed a gradual loss of STAT5 activity during cancer progression [149]. Activated STAT5 was associated with a favorable prognosis in breast cancer patients [150]. Peck et al showed that a loss of pSTAT5 from the nucleus predicts for a poor clinical outcome along 
with the possibility of failure to respond to endocrine therapy in ER+ve breast cancer patients [151]. Interestingly, the expression of Bcl6 is inhibited by prolactin mediated activation of STAT5 in breast cancer [152]. Again, it has been shown that the STAT3 and STAT5 signaling pathway is integrally involved in endocrine resistance and more so in the growth factor-stimulated cases [153].

Thus, there appears to be a fine interplay of functional roles between STAT3 and STAT5 in the various breast cancer subtypes. STAT5 expression corresponds to better prognosis for patient survival by an increase in epithelial cell differentiation and delaying metastasis [154].

Another aspect of STAT5 biology concerns its specificity for a specific breast cancer subtype and its role in endocrine resistance. In premenopausal women which are predominantly hormone responsive tumors or ER+ve tumors, increase in prolaction was associated with increased cancer rick [155]. STAT5 was found to be constitutively activated in ER+ ve tumours [156], and mice studies went onto demonstrate that CA-STAT5 led to the development of tumors in the mammary gland [157].

In 2012, Adrian Britschgi et al demostarted the fine cross talk between signalling pathways and that suppressing the PI3K/mTor pathway, led to an activation of the JAK2/STAT5 pathway and circumvented the efficancy of the PI3K/mTor inhibition [158]. However, dual inhibition of the PI3K/mTor pathway led to a robust response with cancer cell apoptosis and reduction in tumor growth, leading to an increase in the overall survival. This strategy of combined targeting is thus a strategy to combat triple-negative breast cancer (which is characterized by the absence of expression of estrogen and progesterone as well as ERBB2/HER2 receptors), a particularly aggressive and currently incurable disease.

There remains no doubt with the conflicting studies in mice, BC cell lines and human tumour samples, that STAT5 plays a complex role in breast cancer. It appears to have a dual role dependant on the stage of tumour preogression- promoting tumour progression in the early stages of tumour formation while inhibiting their potential to metastasize. It could also be argued that STAT5A/B plays conflicting roles in human and mouse cancer tumorigenesis. Tang et al, on a study in BC cell lines, have reported the differential activity of STAT5a and STAT5b in inducing survival and inbiting cell migration [159]. 
Additionally, new mouse models need to be created and studied to delineate the role of STAT5 in BC. There is now a demand to create models where STAT5 could be knocked out or overexpressed in the tumour once they are formed, so as to help study its role in later stages of cancer progression. Another caveat to studies in breast cancer lies in the various subtypes of breast cancer and the complexity of the developmental process involved in the mammary gland. The role of STAT5 is thus context specific and targeting STAT5 could not only be beneficial in a subset of the breast cancer cell types but also on the stage of the tumour development/progression and as a combination drug.

\section{Conclusion and future prospects}

The JAK-STAT5 pathway has been associated with proliferation, survival, differentiation as well as apoptosis. Activation of STAT5 is dependent on the phosphorylation, acetylation, SUMoylation as well as ubiquitination states. The importance of tetramerization for normal immune functions is well understood and several cancers have shown a dependence on the cancer-promoting actions of STAT5.

While hematopoietic malignancies have been studied in some detail, it is the role of STAT5 in solid cancers and tumours, its regulation of the immune system and the anti-tumour response elicited which warrants further research. In brief, STAT5, by expanding regulatory $\mathrm{T}$ cells which promote tumours and cancers by inhibiting anti-tumour activity, leads to the progression of cancers and tumours. This very role of STAT5 could be exploited for its use as a therapeutic target.

Indeed, several small molecule drugs targeting the cytokine signalling as well as JAK inhibitors have shown promise. Discovery of small molecule therapeutic drugs to target 
STAT5 are in progress, although a STAT5 specific inhibitor is not yet available for use in clinical trials. The challenge to effectively target the JAK-STAT5 pathway for cancer therapy remains and needs extensive research and study.

\section{Acknowledgements}

We thank Dr Susan John (Department of Immunobiology, Kings College London, London, UK) and Dr Stipo Jurcevic for discussions.

\section{REFERENCES}

1. Levy, D.E. and J.E. Darnell, Jr., Stats: transcriptional control and biological impact. Nat Rev Mol Cell Biol, 2002. 3(9): p. 651-62.

2. Aaronson, D.S. and C.M. Horvath, A road map for those who don't know JAK-STAT. Science, 2002. 296(5573): p. 1653-5.

3. Darnell, J.E., Jr., I.M. Kerr, and G.R. Stark, Jak-STAT pathways and transcriptional activation in response to IFNs and other extracellular signaling proteins. Science, 1994. 264(5164): p. 1415-21.

4. Meyer, T., et al., A single residue modulates tyrosine dephosphorylation, oligomerization, and nuclear accumulation of stat transcription factors. J Biol Chem, 2004. 279(18): p. 18998-9007.

5. Marg, A., et al., Nucleocytoplasmic shuttling by nucleoporins Nup153 and Nup214 and CRM1-dependent nuclear export control the subcellular distribution of latent Stat1. J Cell Biol, 2004. 165(6): p. 823-33.

6. Brown, S. and M.P. Zeidler, Unphosphorylated STATs go nuclear. Curr Opin Genet Dev, 2008. 18(5): p. 455-60.

7. Darnell, J.E., Jr., STATs and gene regulation. Science, 1997. 277(5332): p. 1630-5.

8. Copeland, N.G., et al., Distribution of the mammalian Stat gene family in mouse chromosomes. Genomics, 1995. 29(1): p. 225-8.

9. O'Shea, J.J., M. Gadina, and R.D. Schreiber, Cytokine signaling in 2002: new surprises in the Jak/Stat pathway. Cell, 2002. 109 Suppl: p. S121-31.

10. Brivanlou, A.H. and J.E. Darnell, Jr., Signal transduction and the control of gene expression. Science, 2002. 295(5556): p. 813-8.

11. Kisseleva, T., et al., Signaling through the JAK/STAT pathway, recent advances and future challenges. Gene, 2002. 285(1-2): p. 1-24.

12. Leung, S., X. Li, and G.R. Stark, STATs find that hanging together can be stimulating. Science, 1996. 273(5276): p. 750-1.

13. O'Shea, J.J., et al., Jaks and stats as therapeutic targets. Ann Rheum Dis, 2000. 59

Suppl 1: p. i115-8. 
14. Vinkemeier, U., et al., DNA binding of in vitro activated Stat1 alpha, Statl beta and truncated Stat1: interaction between NH2-terminal domains stabilizes binding of two dimers to tandem DNA sites. EMBO J, 1996. 15(20): p. 5616-26.

15. John, S., et al., The significance of tetramerization in promoter recruitment by Stat5. Mol Cell Biol, 1999. 19(3): p. 1910-8.

16. Vinkemeier, U., et al., Structure of the amino-terminal protein interaction domain of STAT-4. Science, 1998. 279(5353): p. 1048-52.

17. Zhang, X. and J.E. Darnell, Jr., Functional importance of Stat3 tetramerization in activation of the alpha 2-macroglobulin gene. J Biol Chem, 2001. 276(36): p. 3357681.

18. Xu, B.C., et al., Growth hormone promotes the association of transcription factor STAT5 with the growth hormone receptor. J Biol Chem, 1996. 271(33): p. 19768-73.

19. Murphy, T.L., et al., Role of the Stat4 $N$ domain in receptor proximal tyrosine phosphorylation. Mol Cell Biol, 2000. 20(19): p. 7121-31.

20. Chen, X., et al., A reinterpretation of the dimerization interface of the $N$-terminal domains of STATs. Protein Sci, 2003. 12(2): p. 361-5.

21. Shuai, K., Modulation of STAT signaling by STAT-interacting proteins. Oncogene, 2000. 19(21): p. 2638-44.

22. Zhang, J.J., et al., Two contact regions between Statl and CBP/p300 in interferon gamma signaling. Proc Natl Acad Sci U S A, 1996. 93(26): p. 15092-6.

23. Becker, S., B. Groner, and C.W. Muller, Three-dimensional structure of the Stat3beta homodimer bound to DNA. Nature, 1998. 394(6689): p. 145-51.

24. Sato, N., et al., Nuclear retention of STAT3 through the coiled-coil domain regulates its activity. Biochem Biophys Res Commun, 2005. 336(2): p. 617-24.

25. Bhattacharya, S. and C. Schindler, Regulation of Stat 3 nuclear export. J Clin Invest, 2003. 111(4): p. 553-9.

26. Horvath, C.M., Z. Wen, and J.E. Darnell, Jr., A STAT protein domain that determines DNA sequence recognition suggests a novel DNA-binding domain. Genes Dev, 1995. 9(8): p. 984-94.

27. Seidel, H.M., et al., Spacing of palindromic half sites as a determinant of selective STAT (signal transducers and activators of transcription) DNA binding and transcriptional activity. Proc Natl Acad Sci U S A, 1995. 92(7): p. 3041-5.

28. Mikita, T., et al., Requirements for interleukin-4-induced gene expression and functional characterization of Stat6. Mol Cell Biol, 1996. 16(10): p. 5811-20.

29. Decker, T., et al., IFNs and STATs in innate immunity to microorganisms. J Clin Invest, 2002. 109(10): p. 1271-7.

30. Ihle, J.N., STATs and MAPKs: obligate or opportunistic partners in signaling. Bioessays, 1996. 18(2): p. 95-8.

31. Decker, T. and P. Kovarik, Serine phosphorylation of STATs. Oncogene, 2000. 19(21): p. 2628-37.

32. Pesu, M., et al., Interleukin-4-induced transcriptional activation by stat6 involves multiple serine/threonine kinase pathways and serine phosphorylation of stat6. Blood, 2000. 95(2): p. 494-502.

33. Bowman, T., et al., STATs in oncogenesis. Oncogene, 2000. 19(21): p. 2474-88.

34. Bhattacharya, S., et al., Cooperation of Stat2 and p300/CBP in signalling induced by interferon-alpha. Nature, 1996. 383(6598): p. 344-7.

35. Zhu, M., et al., Functional association of Nmi with Stat5 and Stat1 in IL-2- and IFNgamma-mediated signaling. Cell, 1999. 96(1): p. 121-30. 
36. Gingras, S., et al., p300/CBP is required for transcriptional induction by interleukin-4 and interacts with Stat6. Nucleic Acids Res, 1999. 27(13): p. 2722-9.

37. Snyder, M., W. He, and J.J. Zhang, The DNA replication factor MCM5 is essential for Stat1-mediated transcriptional activation. Proc Natl Acad Sci U S A, 2005. 102(41): p. 14539-44.

38. Look, D.C., et al., Stat1 depends on transcriptional synergy with Sp1. J Biol Chem, 1995. 270(51): p. 30264-7.

39. Schaefer, T.S., L.K. Sanders, and D. Nathans, Cooperative transcriptional activity of Jun and Stat3 beta, a short form of Stat3. Proc Natl Acad Sci U S A, 1995. 92(20): p. 9097-101.

40. Muhlethaler-Mottet, A., et al., Activation of the MHC class II transactivator CIITA by interferon-gamma requires cooperative interaction between Stat1 and USF-1. Immunity, 1998. 8(2): p. 157-66.

41. Wakao, H., F. Gouilleux, and B. Groner, Mammary gland factor $(M G F)$ is a novel member of the cytokine regulated transcription factor gene family and confers the prolactin response. Embo J, 1994. 13(9): p. 2182-91.

42. Mui, A.L., et al., Interleukin-3, granulocyte-macrophage colony-stimulating factor, and interleukin-5 transduce signals through two forms of STAT5. J Leukoc Biol, 1995. 57(5): p. 799-803.

43. Liu, X., et al., Cloning and expression of Stat5 and an additional homologue (Stat5b) involved in prolactin signal transduction in mouse mammary tissue. Proc Natl Acad Sci U S A, 1995. 92(19): p. 8831-5.

44. Liu, X., et al., Stat5a is mandatory for adult mammary gland development and lactogenesis. Genes Dev, 1997. 11(2): p. 179-86.

45. Horvath, C.M., STAT proteins and transcriptional responses to extracellular signals. Trends Biochem Sci, 2000. 25(10): p. 496-502.

46. Azam, M., et al., Interleukin-3 signals through multiple isoforms of Stat5. Embo J, 1995. 14(7): p. 1402-11.

47. Takeda, K. and S. Akira, STAT family of transcription factors in cytokine-mediated biological responses. Cytokine Growth Factor Rev, 2000. 11(3): p. 199-207.

48. Groner, B., et al., Regulation of the trans-activation potential of STAT5 through its DNA-binding activity and interactions with heterologous transcription factors. Growth Horm IGF Res, 2000. 10 Suppl B: p. S15-20.

49. Van Etten, R.A., Aberrant cytokine signaling in leukemia. Oncogene, 2007. 26(47): p. 6738-49.

50. Schindler, C., et al., Interferon-dependent tyrosine phosphorylation of a latent cytoplasmic transcription factor. Science, 1992. 257(5071): p. 809-13.

51. Shuai, K., et al., Activation of transcription by IFN-gamma: tyrosine phosphorylation of a 91-kD DNA binding protein. Science, 1992. 258(5089): p. 1808-12.

52. Kazansky, A.V., et al., Differential effects of prolactin and src/abl kinases on the nuclear translocation of STAT5B and STAT5A. J Biol Chem, 1999. 274(32): p. 22484-92.

53. Hayakawa, F. and T. Naoe, SFK-STAT pathway: an alternative and important way to malignancies. Ann N Y Acad Sci, 2006. 1086: p. 213-22.

54. Aoki, N. and T. Matsuda, A nuclear protein tyrosine phosphatase TC-PTP is a potential negative regulator of the PRL-mediated signaling pathway: dephosphorylation and deactivation of signal transducer and activator of transcription $5 a$ and 5 b by TC-PTP in nucleus. Mol Endocrinol, 2002. 16(1): p. 5869. 
55. Yokoyama, N., N.C. Reich, and W.T. Miller, Involvement of protein phosphatase $2 A$ in the interleukin-3-stimulated Jak2-Stat5 signaling pathway. J Interferon Cytokine Res, 2001. 21(6): p. 369-78.

56. Chen, Y., et al., Identification of Shp-2 as a Stat5A phosphatase. J Biol Chem, 2003. 278(19): p. 16520-7.

57. Aoki, N. and T. Matsuda, A cytosolic protein-tyrosine phosphatase PTP1B specifically dephosphorylates and deactivates prolactin-activated STAT5a and STAT5b. J Biol Chem, 2000. 275(50): p. 39718-26.

58. $\mathrm{Xu}, \mathrm{D}$. and C.K. Qu, Protein tyrosine phosphatases in the JAK/STAT pathway. Front Biosci, 2008. 13: p. 4925-32.

59. Hoyt, R., et al., Cutting edge: selective tyrosine dephosphorylation of interferonactivated nuclear STAT5 by the VHR phosphatase. J Immunol, 2007. 179(6): p. 34026.

60. Rathinam, C., et al., The E3 ubiquitin ligase c-Cbl restricts development and functions of hematopoietic stem cells. Genes Dev, 2008. 22(8): p. 992-7.

61. Chen, Y., et al., Proteasome-dependent down-regulation of activated Stat5A in the nucleus. Blood, 2006. 108(2): p. 566-74.

62. Yamashita, H., et al., Differential control of the phosphorylation state of prolinejuxtaposed serine residues Ser725 of Stat5a and Ser730 of Stat5b in prolactinsensitive cells. J Biol Chem, 1998. 273(46): p. 30218-24.

63. Yamashita, H., et al., Role of serine phosphorylation of Stat5a in prolactin-stimulated beta-casein gene expression. Mol Cell Endocrinol, 2001. 183(1-2): p. 151-63.

64. Wang, R.A., et al., Essential functions of p21-activated kinase 1 in morphogenesis and differentiation of mammary glands. J Cell Biol, 2003. 161(3): p. 583-92.

65. Clark, D.E., et al., ERBB4/HER4 potentiates STAT5A transcriptional activity by regulating novel STAT5A serine phosphorylation events. J Biol Chem, 2005. 280(25): p. 24175-80.

66. Friedbichler, K., et al., Stat5a serine 725 and 779 phosphorylation is a prerequisite for hematopoietic transformation. Blood, 2010. 116(9): p. 1548-58.

67. Berger, A., et al., PAK-dependent STAT5 serine phosphorylation is required for BCRABL-induced leukemogenesis. Leukemia, 2014. 28(3): p. 629-41.

68. Gewinner, C., et al., The coactivator of transcription CREB-binding protein interacts preferentially with the glycosylated form of Stat5. J Biol Chem, 2004. 279(5): p. 3563-72.

69. Nanashima, N., et al., Nuclear localization of STAT5A modified with O-linked Nacetylglucosamine and early involution in the mammary gland of Hirosaki hairless rat. J Biol Chem, 2005. 280(52): p. 43010-6.

70. Jackson, P.K., A new RING for SUMO: wrestling transcriptional responses into nuclear bodies with PIAS family E3 SUMO ligases. Genes Dev, 2001. 15(23): p. 3053-8.

71. Mziaut, H., et al., Synergy of glucose and growth hormone signalling in islet cells through ICA512 and STAT5. Nat Cell Biol, 2006. 8(5): p. 435-45.

72. Van Nguyen, T., et al., SUMO-specific protease 1 is critical for early lymphoid development through regulation of STAT5 activation. Mol Cell, 2012. 45(2): p. 21021.

73. Rycyzyn, M.A. and C.V. Clevenger, The intranuclear prolactin/cyclophilin B complex as a transcriptional inducer. Proc Natl Acad Sci U S A, 2002. 99(10): p. 6790-5. 
74. Dieudonne, F.X., et al., Promotion of osteoblast differentiation in mesenchymal cells through Cbl-mediated control of STAT5 activity. Stem Cells, 2013. 31(7): p. 1340-9.

75. Pfitzner, E., et al., p300/CREB-binding protein enhances the prolactin-mediated transcriptional induction through direct interaction with the transactivation domain of Stat5, but does not participate in the Stat5-mediated suppression of the glucocorticoid response. Mol Endocrinol, 1998. 12(10): p. 1582-93.

76. Litterst, C.M., et al., NCoA-1/SRC-1 is an essential coactivator of STAT5 that binds to the FDL motif in the alpha-helical region of the STAT5 transactivation domain. J Biol Chem, 2003. 278(46): p. 45340-51.

77. Engblom, D., et al., Direct glucocorticoid receptor-Stat5 interaction in hepatocytes controls body size and maturation-related gene expression. Genes Dev, 2007. 21(10): p. $1157-62$.

78. Stocklin, E., et al., Functional interactions between Stat5 and the glucocorticoid receptor. Nature, 1996. 383(6602): p. 726-8.

79. Chen, X., et al., Crystal structure of a tyrosine phosphorylated STAT-1 dimer bound to DNA. Cell, 1998. 93(5): p. 827-39.

80. Verdier, F., et al., A sequence of the CIS gene promoter interacts preferentially with two associated STAT5A dimers: a distinct biochemical difference between STAT5A and STAT5B. Mol Cell Biol, 1998. 18(10): p. 5852-60.

81. Meyer, W.K., et al., Interaction of STAT5 dimers on two low affinity binding sites mediates interleukin 2 (IL-2) stimulation of IL-2 receptor alpha gene transcription. $\mathrm{J}$ Biol Chem, 1997. 272(50): p. 31821-8.

82. Kirken, R.A., et al., Prolactin stimulates serine/tyrosine phosphorylation and formation of heterocomplexes of multiple Stat5 isoforms in Nb2 lymphocytes. J Biol Chem, 1997. 272(22): p. 14098-103.

83. Park, S.H. and D.J. Waxman, Inhibitory cross-talk between STAT5b and liver nuclear factor HNF3beta: impact on the regulation of growth hormone pulse-stimulated, male-specific liver cytochrome P-450 gene expression. J Biol Chem, 2001. 276(46): p. 43031-9.

84. Meinke, A., et al., Activation of different Stat5 isoforms contributes to cell-typerestricted signaling in response to interferons. Mol Cell Biol, 1996. 16(12): p. 693744.

85. Boucheron, C., et al., A single amino acid in the DNA binding regions of STAT5A and STAT5B confers distinct DNA binding specificities. J Biol Chem, 1998. 273(51): p. 33936-41.

86. Soldaini, E., et al., DNA binding site selection of dimeric and tetrameric Stat5 proteins reveals a large repertoire of divergent tetrameric Stat5a binding sites. Mol Cell Biol, 2000. 20(1): p. 389-401.

87. Ehret, G.B., et al., DNA binding specificity of different STAT proteins. Comparison of in vitro specificity with natural target sites. J Biol Chem, 2001. 276(9): p. 6675-88.

88. Lin, J.X. and W.J. Leonard, The role of Stat5a and Stat5b in signaling by IL-2 family cytokines. Oncogene, 2000. 19(21): p. 2566-76.

89. Schindler, C. and I. Strehlow, Cytokines and STAT signaling. Adv Pharmacol, 2000. 47: p. 113-74.

90. Fung, M.M., et al., IL-2- and STAT5-regulated cytokine gene expression in cells expressing the Tax protein of HTLV-1. Oncogene, 2005. 24(29): p. 4624-33.

91. Moriggl, R., et al., Stat5 tetramer formation is associated with leukemogenesis. Cancer Cell, 2005. 7(1): p. 87-99. 
92. Lin, J.X., et al., Critical Role of STAT5 transcription factor tetramerization for cytokine responses and normal immune function. Immunity, 2012. 36(4): p. 586-99.

93. Udy, G.B., et al., Requirement of STAT5b for sexual dimorphism of body growth rates and liver gene expression. Proc Natl Acad Sci U S A, 1997. 94(14): p. 7239-44.

94. Teglund, S., et al., Stat5a and Stat5b proteins have essential and nonessential, or redundant, roles in cytokine responses. Cell, 1998. 93(5): p. 841-50.

95. Cui, Y., et al., Inactivation of Stat5 in mouse mammary epithelium during pregnancy reveals distinct functions in cell proliferation, survival, and differentiation. Mol Cell Biol, 2004. 24(18): p. 8037-47.

96. Moriggl, R., et al., Stat5 is required for IL-2-induced cell cycle progression of peripheral T cells. Immunity, 1999. 10(2): p. 249-59.

97. Lecine, P., et al., Elf-1 and Stat5 bind to a critical element in a new enhancer of the human interleukin-2 receptor alpha gene. Mol Cell Biol, 1996. 16(12): p. 6829-40.

98. Nakajima, H., et al., An indirect effect of Stat5a in IL-2-induced proliferation: a critical role for Stat5a in IL-2-mediated IL-2 receptor alpha chain induction. Immunity, 1997. 7(5): p. 691-701.

99. Imada, K., et al., Stat5b is essential for natural killer cell-mediated proliferation and cytolytic activity. J Exp Med, 1998. 188(11): p. 2067-74.

100. Snow, J.W., et al., STAT5 promotes multilineage hematolymphoid development in vivo through effects on early hematopoietic progenitor cells. Blood, 2002. 99(1): p. 95-101.

101. Kelly, J., et al., A role for Stat5 in CD8+ T cell homeostasis. J Immunol, 2003. 170(1): p. 210-7.

102. Kagami, S., et al., Both stat5a and stat5b are required for antigen-induced eosinophil and T-cell recruitment into the tissue. Blood, 2000. 95(4): p. 1370-7.

103. Friedrich, K., et al., Activation of STAT5 by IL-4 relies on Janus kinase function but not on receptor tyrosine phosphorylation, and can contribute to both cell proliferation and gene regulation. Int Immunol, 1999. 11(8): p. 1283-94.

104. Sexl, V., et al., Stat5a/b contribute to interleukin 7-induced B-cell precursor expansion, but abl-and bcr/abl-induced transformation are independent of stat5. Blood, 2000. 96(6): p. 2277-83.

105. Hoelbl, A., et al., Clarifying the role of Stat5 in lymphoid development and Abelsoninduced transformation. Blood, 2006. 107(12): p. 4898-906.

106. Yao, Z., et al., Stat5a/b are essential for normal lymphoid development and differentiation. Proc Natl Acad Sci U S A, 2006. 103(4): p. 1000-5.

107. Park, J.H., et al., 'Coreceptor tuning': cytokine signals transcriptionally tailor CD8 coreceptor expression to the self-specificity of the TCR. Nat Immunol, 2007. 8(10): p. 1049-59.

108. Zhu, J. and W.E. Paul, Peripheral CD4+ T-cell differentiation regulated by networks of cytokines and transcription factors. Immunol Rev, 2010. 238(1): p. 247-62.

109. Zhu, J., et al., Stat5 activation plays a critical role in Th2 differentiation. Immunity, 2003. 19(5): p. 739-48.

110. Liao, W., et al., Priming for T helper type 2 differentiation by interleukin 2-mediated induction of interleukin 4 receptor alpha-chain expression. Nat Immunol, 2008. 9(11): p. 1288-96.

111. Liao, W., et al., Modulation of cytokine receptors by IL-2 broadly regulates differentiation into helper T cell lineages. Nat Immunol, 2011. 12(6): p. 551-9.

112. Burchill, M.A., et al., IL-2 receptor beta-dependent STAT5 activation is required for the development of Foxp3+ regulatory T cells. J Immunol, 2007. 178(1): p. 280-90. 
113. Shuai, K., et al., Constitutive activation of STAT5 by the BCR-ABL oncogene in chronic myelogenous leukemia. Oncogene, 1996. 13(2): p. 247-54.

114. Lin, T.S., S. Mahajan, and D.A. Frank, STAT signaling in the pathogenesis and treatment of leukemias. Oncogene, 2000. 19(21): p. 2496-504.

115. Ilaria, R.L., Jr. and R.A. Van Etten, P210 and P190(BCR/ABL) induce the tyrosine phosphorylation and DNA binding activity of multiple specific STAT family members. J Biol Chem, 1996. 271(49): p. 31704-10.

116. Van Etten, R.A., Oncogenic signaling: new insights and controversies from chronic myeloid leukemia. J Exp Med, 2007. 204(3): p. 461-5.

117. Buettner, R., L.B. Mora, and R. Jove, Activated STAT signaling in human tumors provides novel molecular targets for therapeutic intervention. Clin Cancer Res, 2002. 8(4): p. 945-54.

118. Bromberg, J., Stat proteins and oncogenesis. J Clin Invest, 2002. 109(9): p. 1139-42.

119. Mitchell, T.J., S.J. Whittaker, and S. John, Dysregulated expression of COOHterminally truncated Stat5 and loss of IL2-inducible Stat5-dependent gene expression in Sezary Syndrome. Cancer Res, 2003. 63(24): p. 9048-54.

120. Xia, Z., et al., Truncated STAT proteins are prevalent at relapse of acute myeloid leukemia. Leuk Res, 2001. 25(6): p. 473-82.

121. Nakayama, J., et al., BLNK suppresses pre-B-cell leukemogenesis through inhibition of JAK3. Blood, 2009. 113(7): p. 1483-92.

122. Joliot, V., et al., Constitutive STAT5 activation specifically cooperates with the loss of p53 function in B-cell lymphomagenesis. Oncogene, 2006. 25(33): p. 4573-84.

123. Weber-Nordt, R.M., et al., Constitutive activation of STAT proteins in primary lymphoid and myeloid leukemia cells and in Epstein-Barr virus (EBV)-related lymphoma cell lines. Blood, 1996. 88(3): p. 809-16.

124. Gouilleux-Gruart, V., et al., Activated Stat related transcription factors in acute leukemia. Leuk Lymphoma, 1997. 28(1-2): p. 83-8.

125. Ye, D., et al., STAT5 signaling is required for the efficient induction and maintenance of CML in mice. Blood, 2006. 107(12): p. 4917-25.

126. Schaller-Schonitz, M., et al., BCR-ABL affects STAT5A and STAT5B differentially. PLoS One, 2014. 9(5): p. e97243.

127. Casetti, L., et al., Differential contributions of STAT5A and STAT5B to stress protection and tyrosine kinase inhibitor resistance of chronic myeloid leukemia stem/progenitor cells. Cancer Res, 2013. 73(7): p. 2052-8.

128. Hochhaus, A. and P. La Rosee, Imatinib therapy in chronic myelogenous leukemia: strategies to avoid and overcome resistance. Leukemia, 2004. 18(8): p. 1321-31.

129. Onishi, M., et al., Identification and characterization of a constitutively active STAT5 mutant that promotes cell proliferation. Mol Cell Biol, 1998. 18(7): p. 3871-9.

130. Baxter, E.J., et al., Acquired mutation of the tyrosine kinase JAK2 in human myeloproliferative disorders. Lancet, 2005. 365(9464): p. 1054-61.

131. Levine, R.L., et al., Activating mutation in the tyrosine kinase JAK2 in polycythemia vera, essential thrombocythemia, and myeloid metaplasia with myelofibrosis. Cancer Cell, 2005. 7(4): p. 387-97.

132. Germeshausen, M., M. Ballmaier, and K. Welte, Incidence of CSF3R mutations in severe congenital neutropenia and relevance for leukemogenesis: Results of a longterm survey. Blood, 2007. 109(1): p. 93-9.

133. Wernig, G., et al., Expression of Jak2 V617F causes a polycythemia vera-like disease with associated myelofibrosis in a murine bone marrow transplant model. Blood, 2006. 107(11): p. 4274-81. 
134. Taketani, T., et al., FLT3 mutations in the activation loop of tyrosine kinase domain are frequently found in infant ALL with MLL rearrangements and pediatric ALL with hyperdiploidy. Blood, 2004. 103(3): p. 1085-8.

135. Mizuki, M., et al., Oncogenic receptor tyrosine kinase in leukemia. Cell Mol Biol (Noisy-le-grand), 2003. 49(6): p. 907-22.

136. Liu, X., G.W. Robinson, and L. Hennighausen, Activation of Stat5a and Stat5b by tyrosine phosphorylation is tightly linked to mammary gland differentiation. Mol Endocrinol, 1996. 10(12): p. 1496-506.

137. Miyoshi, K., et al., Signal transducer and activator of transcription (Stat) 5 controls the proliferation and differentiation of mammary alveolar epithelium. J Cell Biol, 2001. 155(4): p. 531-42.

138. Tourkine, N., et al., Activation of STAT factors by prolactin, interferon-gamma, growth hormones, and a tyrosine phosphatase inhibitor in rabbit primary mammary epithelial cells. J Biol Chem, 1995. 270(36): p. 20952-61.

139. Chughtai, N., et al., Prolactin induces SHP-2 association with Stat5, nuclear translocation, and binding to the beta-casein gene promoter in mammary cells. J Biol Chem, 2002. 277(34): p. 31107-14.

140. Tan, S.H. and M.T. Nevalainen, Signal transducer and activator of transcription 5A/B in prostate and breast cancers. Endocr Relat Cancer, 2008. 15(2): p. 367-90.

141. Humphreys, R.C. and L. Hennighausen, Signal transducer and activator of transcription 5 a influences mammary epithelial cell survival and tumorigenesis. Cell Growth Differ, 1999. 10(10): p. 685-94.

142. Ren, S., et al., Loss of Stat5a delays mammary cancer progression in a mouse model. Oncogene, 2002. 21(27): p. 4335-9.

143. Iavnilovitch, E., et al., Deregulation of Stat5 expression and activation causes mammary tumors in transgenic mice. Int J Cancer, 2004. 112(4): p. 607-19.

144. Vafaizadeh, V., et al., Mammary epithelial reconstitution with gene-modified stem cells assigns roles to Stat5 in luminal alveolar cell fate decisions, differentiation, involution, and mammary tumor formation. Stem Cells, 2010. 28(5): p. 928-38.

145. Yamashita, H., et al., Naturally occurring dominant-negative Stat5 suppresses transcriptional activity of estrogen receptors and induces apoptosis in T47D breast cancer cells. Oncogene, 2003. 22(11): p. 1638-52.

146. Caffarel, M.M., et al., Constitutive activation of JAK2 in mammary epithelium elevates Stat5 signalling, promotes alveologenesis and resistance to cell death, and contributes to tumourigenesis. Cell Death Differ, 2012. 19(3): p. 511-22.

147. Walker, S.R., M. Xiang, and D.A. Frank, Distinct roles of STAT3 and STAT5 in the pathogenesis and targeted therapy of breast cancer. Mol Cell Endocrinol, 2014. 382(1): p. 616-21.

148. Yamashita, H., et al., Stat5 expression predicts response to endocrine therapy and improves survival in estrogen receptor-positive breast cancer. Endocr Relat Cancer, 2006. 13(3): p. 885-93.

149. Sultan, A.S., et al., Stat5 promotes homotypic adhesion and inhibits invasive characteristics of human breast cancer cells. Oncogene, 2005. 24(5): p. 746-60.

150. Nevalainen, M.T., et al., Signal transducer and activator of transcription-5 activation and breast cancer prognosis. J Clin Oncol, 2004. 22(11): p. 2053-60.

151. Peck, A.R., et al., Loss of nuclear localized and tyrosine phosphorylated Stat5 in breast cancer predicts poor clinical outcome and increased risk of antiestrogen therapy failure. J Clin Oncol, 2011. 29(18): p. 2448-58. 
152. Tran, T.H., et al., Prolactin inhibits BCL6 expression in breast cancer through a Stat5a-dependent mechanism. Cancer Res, 2010. 70(4): p. 1711-21.

153. Silva, C.M. and M.A. Shupnik, Integration of steroid and growth factor pathways in breast cancer: focus on signal transducers and activators of transcription and their potential role in resistance. Mol Endocrinol, 2007. 21(7): p. 1499-512.

154. Wagner, K.U. and H. Rui, Jak2/Stat5 signaling in mammogenesis, breast cancer initiation and progression. J Mammary Gland Biol Neoplasia, 2008. 13(1): p. 93-103.

155. Tworoger, S.S., P. Sluss, and S.E. Hankinson, Association between plasma prolactin concentrations and risk of breast cancer among predominately premenopausal women. Cancer Res, 2006. 66(4): p. 2476-82.

156. Cotarla, I., et al., Stat5a is tyrosine phosphorylated and nuclear localized in a high proportion of human breast cancers. Int J Cancer, 2004. 108(5): p. 665-71.

157. Iavnilovitch, E., B. Groner, and I. Barash, Overexpression and forced activation of stat5 in mammary gland of transgenic mice promotes cellular proliferation, enhances differentiation, and delays postlactational apoptosis. Mol Cancer Res, 2002. 1(1): p. 32-47.

158. Britschgi, A., et al., JAK2/STAT5 inhibition circumvents resistance to PI3K/mTOR blockade: a rationale for cotargeting these pathways in metastatic breast cancer. Cancer Cell, 2012. 22(6): p. 796-811.

159. Tang, J.Z., et al., Signal transducer and activator of transcription (STAT)-5A and STAT5B differentially regulate human mammary carcinoma cell behavior. Endocrinology, 2010. 151(1): p. 43-55.

160. Yu, H., D. Pardoll, and R. Jove, STATs in cancer inflammation and immunity: a leading role for STAT3. Nat Rev Cancer, 2009. 9(11): p. 798-809.

161. Schindler, C.W., Series introduction. JAK-STAT signaling in human disease. J Clin Invest, 2002. 109(9): p. 1133-7.

162. Leonard, W.J., Role of Jak kinases and STATs in cytokine signal transduction. Int J Hematol, 2001. 73(3): p. 271-7.

163. Rane, S.G. and E.P. Reddy, JAKs, STATs and Src kinases in hematopoiesis. Oncogene, 2002. 21(21): p. 3334-58.

164. Barash, I., Stat5 in the mammary gland: controlling normal development and cancer. J Cell Physiol, 2006. 209(2): p. 305-13. 
Table 1: Effector molecules and the kinases involved in signalling of STAT5

\begin{tabular}{ll}
\hline Ligands & JAK's and non JAK kinases \\
\hline IL-2 & JAK1, JAK3, Fyn, Lck, Hck, Tec, \\
IL-3 & JAK2, Fyn, Hck, Lyn \\
IL-4 & JAK1, JAK3 \\
IL-5 & JAK2, Btk \\
IL-7 & JAK1, JAK3, Lyn \\
IL-9 & JAK3 \\
IL-10 & JAK1, Tyk2 \\
IL-15 & JAK1, Lck \\
IL-21 & JAK3 \\
IL-22 & JAK1, Tyk2 \\
IL-27 & JAK1, JAK2, Tyk2 \\
EGF & JAK1, EGFR, Src \\
EPO & JAK2, Src Family \\
GH & JAK2, Src Family \\
GM-CSF & JAK2, Hck, Lyn \\
Insulin & JAK2, IR, Src \\
Leptin & JAK2 \\
PDGF & JAK1, PDGFR, Src \\
PRL & JAK2, Src \\
TPO & Tyk2, JAK2, Lyn \\
\hline
\end{tabular}


Abbreviations: EGF, epidermal growth factor; EPO, erythropoietin; GH, growth hormone; GM-CSF, granulocyte macrophage-colony stimulating factor); PDGF, platelet-derived growth factor; PRL, prolactin; TPO, thrombopoietin; IL, interleukin; JAK, Janus kinase; STAT, signal transduction and activation of transcription; TYK, tyrosine kinase. Compiled from [161-163].

Table 2: Protein interaction partners of STAT5

\begin{tabular}{ll}
\hline Protein & Interacting domain \\
\hline BRCA1 and BRCA2 & not determined \\
Caveolin & not determined \\
transactivation domain of STAT5a & SH2 domain of STAT5 \\
CrkL & SH2 domain of CrkL \\
ERK & transactivation domain of STAT5a \\
ER $\alpha$ & transactivation domain of STAT5 \\
Ligand/CypB & N terminus of STAT5 \\
NcoA-1/SRC-1 & transactivation domain of STAT5a \\
Nmi & coiled-coil domain of STAT5 \\
Oct1 & transactivation domain of STAT5 \\
p100 & transactivation domain of STAT5 \\
SMRT & coiled-coil domain of STAT5 \\
STAP-2/BKS & transactivation domain of STAT5 \\
TR 31 & non-determined \\
\hline
\end{tabular}

References: [164] 
